\title{
Association of molecular biomarkers heterogeneity and treatment pattern, disease outcomes in multifocal or multicentric breast cancer patients
}

Shuai Li

Shanghai Jiao Tong University Medical School Affiliated Ruijin Hospital Jiayi Wu

Shanghai Jiao Tong University Medical School Affiliated Ruijin Hospital

Ou Huang

Shanghai Jiao Tong University Medical School Affiliated Ruijin Hospital

Jianrong He

Shanghai Jiao Tong University Medical School Affiliated Ruijin Hospital

Li Zhu

Shanghai Jiao Tong University Medical School Affiliated Ruijin Hospital

Weiguo Chen

Shanghai Jiao Tong University Medical School Affiliated Ruijin Hospital

Yafen Li

Shanghai Jiao Tong University Medical School Affiliated Ruijin Hospital

Xiaosong Chen ( $\nabla$ chenxiaosong0156@hotmail.com )

Ruijin Hospital, Shanghai Jiaotong University School of Medicine https://orcid.org/0000-0002-32860035

Kunwei Shen

Shanghai Jiao Tong University Medical School Affiliated Ruijin Hospital

\section{Research Article}

Keywords: Biomarkers, Breast neoplasms, Intratumor heterogeneity, Multifocal, Multicentric, Prognosis

Posted Date: May 27th, 2021

DOI: https://doi.org/10.21203/rs.3.rs-559597/v1

License: (c) (1) This work is licensed under a Creative Commons Attribution 4.0 International License.

Read Full License 


\section{Abstract \\ Purpose}

To evaluate the rates of estrogen receptor (ER), progesterone receptor (PR), human epidermal growth factor receptor 2 (HER2), and Ki-67 heterogeneity in multifocal or multicentric breast cancer (MMBC) and its association with treatment pattern and disease outcomes.

\section{Methods}

MMBC patients with ER, PR, HER2, and Ki67 results for each tumor focus were retrospectively analyzed and categorized into the Homo group and the Hetero group. Chi-square test were performed to compare the treatment options between the groups. Disease-free survival (DFS) and overall survival (OS) rates were estimated from Kaplan-Meier curves and compared between two groups.

\section{Results}

A total of 330 patients were included and $53(16.1 \%)$ were classified into the Hetero group. Adjuvant endocrine therapy was more frequently assigned for patients in the Hetero group than in the Homo group (84.9\% vs. $71.7 \%, P=0.046)$. There was no difference in terms of adjuvant anti-HER2 therapy $(28.3 \%$ vs. $19.6 \%, P=0.196)$ and chemotherapy $(69.9 \%$ vs. $69.8 \%, P=0.987)$ usage between two groups. At a median follow-up of 36 months, DFS rates were $81.2 \%$ for the Hetero group and $96.5 \%$ for the Homo group $(H R=2.81,95 \% \mathrm{Cl}: 1.00-7.88, P=0.041)$. The estimated 3-year OS rates for the groups were $95.8 \%$ and $99.5 \%$, respectively $(H R=4.31,95 \% \mathrm{Cl}: 0.83-22.46, P=0.059)$.

\section{Conclusion}

Heterogeneity of ER, PR, HER2, or Ki67 was present in 16.1\% patients with MMBC. Biomarkers heterogeneity influenced adjuvant endocrine therapy usage and was associated with worse disease outcomes, indicating further clinical evaluation.

\section{Introduction}

Breast cancer is a heterogeneous group of diseases in which individual patient differs in morphological features, molecular profiles, therapeutic responses, and prognosis [1]. Morphological variability such as pathological type and histological grade has been well documented for decades and forms the basis for histological classification of breast cancer. More recently, different molecular phenotypes of breast cancer have been defined by genetic or immunohistochemistry testing. For example, the well-defined 2013 St Gallen subtypes of breast cancer were based on the expressions of estrogen (ER) and 
progesterone (PR) receptors, human epidermal growth factor receptor 2 (HER2), and Ki67 proliferative index, which provide prognostic information and can be used to tailor systemic adjuvant therapy [2].

The molecular heterogeneity can occur either between different patients (intertumor heterogeneity), or within the same patient (intratumor heterogeneity) [1]. Heterogenous expressions of ER, PR, HER2, and Ki67 have been widely reported between core needle biopsy and surgical samples, between different regions of a primary tumor, between a primary tumor and a matched metastatic lesion, or between metastatic lesions [3-9]. Beyond spatial heterogeneity, heterogeneity can be observed as the natural evolution of a tumor or as consequences of anticancer treatments [10-12].

In a previous study that evaluated the heterogeneity of ER, PR, HER2, and Ki67 between different foci in multifocal/multicentric breast cancer (MMBC), the heterogeneity of these molecular markers was present in $4.4 \%, 15.9 \%, 9.7 \%$, and $15.0 \%$ cases [13]. However, there are few studies investigating the therapeutic and prognostic impact of such heterogeneity [13-14]. Herein, we performed this retrospective study to evaluate the rates of ER, PR, HER2, and Ki67 heterogeneity in patients with MMBC as well as its impacts on systemic adjuvant therapy decision-making and disease outcomes.

\section{Methods}

\section{Study population}

Patients who received surgery and were diagnosed with multifocal or multicentric breast cancer at Department of General Surgery, Comprehensive Breast Health Center, Ruijin Hospital, Shanghai Jiao Tong University School of Medicine from Jan 2009 to Dec 2018 were retrospectively analyzed.

Clinicopathological characteristics, adjuvant treatment, and follow-up data were retrieved from Shanghai Jiao Tong University Breast Cancer Database (SJTU-BCDB). The eligibility criteria were as follows: (1) invasive carcinoma for all tumor foci; (2) no distant metastasis at diagnosis; (3) ER, PR, HER2, and Ki67 both tested between different invasive tumor foci. Those who received neo-adjuvant therapy and those with extensive DCIS were excluded from the present study.

\section{Histopathology assessments}

Histopathology analysis for different tumor foci on surgical specimens were independently performed and reviewed by two pathologists at the Department of Pathology, Ruijin Hospital, Shanghai Jiao Tong University School of Medicine [15-16]. In this study, multifocality was defined as the presence of more than one focus of invasive carcinoma in one breast quadrant; and multicentricity was defined as the presence of a focus in a different breast quadrant from the main lesion [13]. Immunohistochemistry (IHC) of ER, PR, HER2 and fluorescence in situ hybridization (FISH) of CerbB-2, if necessary, were performed on surgical specimens according to the ASCO/CAP guidelines [17-20]. In brief, ER/PR were considered positive if a minimum of $1 \%$ of the invasive tumor cells were positive for ER/PR staining and HER2 positivity was defined as IHC $3+$ or IHC 2 + with FISH CerbB-2 amplification. Molecular subtypes were determined based on 2013 St Gallen system: luminal A-like (ER+/PR $\geq 20 \% / H E R 2-/ K i 67<20 \%$ ), luminal 
B-like (HER2-) (ER+/HER2-/Ki67 $\geq 20 \%$ or ER+/PR < 20\%/HER2 or ER-/PR+/HER2-), luminal B-like (HER2+) (ER + or PR+/HER2+), HER2+ (ER-/PR-/HER2+) and triple negative (ER-/PR-/HER2-) [2]. Patients with concordant status of ER, PR, HER2, and Ki67 among all invasive tumor foci were categorized into the Homo group, while the Hetero group was defined as the existence of at least one discordance for ER, PR, HER2, or Ki67 between different foci. The main focus referred to the largest tumor focus and the other foci were named minor foci.

\section{Treatment and follow-up}

Adjuvant treatment decisions were made through multidisciplinary team (MDT) meetings attended by surgical oncologists, medical oncologists, radiation oncologists, and pathologists [21]. The patients were followed up every 3 months during the first two years after surgery, every 6 months from the third to the fifth years, and once per year hereafter till death. DFS was defined as the period from the date of surgery to first local-regional relapse, contralateral breast cancer, secondary new malignant tumor, distant relapse, or death. OS was calculated from the date of surgery to the date of death.

\section{Statistics}

Kappa test were performed to evaluate the concordance rates of pathological type, histological grade, ER, PR, HER2, Ki67, and molecular subtype between the larger tumor focus and the smaller focus. For tumors with 3 or 4 foci, the results were considered concordant only when the biomarkers status of all tumor foci were concordant. The adjuvant therapy options were compared between the Homo group and the Hetero group using chi-square test or Fisher exact test. DFS and OS rates were estimated from Kaplan-Meier curves and compared between the two groups via log-rank test. Cox proportional hazard model was used to calculate the hazard ratios for relapse and death. Two-side $P<0.05$ was considered statistically significant. All the statistical procedures were performed on SPSS (version 26.0).

\section{Results}

\section{Baseline clinicopathological characteristics}

There were 584 women with multifocal or multicentric breast cancers and 330 of them were included in the study (Fig. 1). Physical examination, sonography, mammography, and MRI identified 16.5\%, 68.9\%, $35.7 \%$, and $78.1 \%$ of these patients, respectively (Supplementary Figure S1). As shown in the Supplementary Figure S2, the median distance between the main and minor foci was 12.5 (IQR: 7.1-20.0) $\mathrm{mm}$, which showed no significant difference between the Homo and Hetero groups (12.4 mm versus 14.0 $\mathrm{mm}, P=0.359)$. The demographic and clinicopathologic characteristics for the cohort are summarized in Table 1. The median age for the patients was 56 (IQR: 47-65) years and 43.8\% patients were pre/perimenopausal at diagnosis. Patients with 2 and $3 / 4$ foci accounted for $92.4 \%$ and $7.6 \%$ of the cohort, respectively. Twenty-six (7.9\%) patients received breast-conserving surgery and sentinel lymph node biopsy was performed in 103 (31.5\%) patients. A total of $45.7 \%$ and $41.2 \%$ patients with multifocal and multicentric diseases received radiotherapy, respectively (Supplementary Figure S3). There were 130 
(39.9\%) patients whose main tumor foci were larger than $2.0 \mathrm{~cm}$ and $132(40.0 \%)$ patients had positive axillary lymph nodes (ALN). 
Table 1

Baseline clinical and pathological characteristics of the whole population

\section{Characteristics}

Age $(y / 0)$

Menstrual status

Pre/Peri-

Post-

Number of foci

2

$3 / 4$

Location of foci

Multifocal

Multicentric

Breast surgery

BCS

Mastectomy

Axillary surgery

SLNB

ALND

Pathological type ${ }^{\text {a }}$

IDC

Non-IDC

Tumor size ${ }^{a}$

$\leq 2.0 \mathrm{~cm}$

$>2.0 \mathrm{~cm}$

ALN status
No.

56 (IQR: 47-65)
$\%$

144

43.8

185

56.2

305

92.4

25

7.6

211

63.9

119

36.1

26

7.9

304

92.1

103

31.5

224

68.5

267

80.9

63

19.1

196

60.1

130

a : main focus.

Abbreviations: ALN, axillary lymph node; ALND: axillary lymph node dissection; BCS, breastconserving surgery; IDC: invasive ductal carcinoma; NA: not available; SLNB: sentinel lymph node biopsy; y/o, years old. 


\begin{tabular}{|lll|}
\hline Characteristics & No. & $\%$ \\
\hline Negative & 198 & 60.0 \\
\hline Positive & 132 & 40.0 \\
\hline Histological grade $^{\text {a }}$ & & \\
\hline$\square$ & 20 & 6.1 \\
\hline$\square$ & 160 & 48.5 \\
\hline$\square$ & 83 & 25.2 \\
\hline NA & 67 & 20.3 \\
\hline
\end{tabular}

a : main focus.

Abbreviations: ALN, axillary lymph node; ALND: axillary lymph node dissection; BCS, breastconserving surgery; IDC: invasive ductal carcinoma; NA: not available; SLNB: sentinel lymph node biopsy; y/o, years old.

\section{Rates of molecular markers heterogeneity}

$E R, P R, H E R 2$, and Ki67 mismatches of the main tumor focus between CNB and surgical samples were present in 15 (6.6\%), 32 (14.0\%), 9 (5.5\%), and 57 (25.1\%) patients, respectively (Supplementary Table S1). As shown in Table 2, concordance rates of ER, PR, HER2, and Ki67 among different tumor foci were $97.0 \%, 93.9 \%, 96.5 \%$, and $91.2 \%$, respectively (all $P$ values $<0.001$ ). Heterogeneity of ER, PR, HER2, and Ki67 were present in 10 (3.0\%), 20 (6.1\%), 11 (3.5\%), and 29 (8.8\%) patients, respectively. Among the whole cohort, a total of $53(16.1 \%)$ patients showed intratumor heterogeneity of molecular markers and the remaining 277 (83.9\%) were homogenous. 
Table 2

Concordance rates of pathological type, histological grade, ER, PR, HER2, and Ki67 status

\begin{tabular}{|c|c|c|c|c|c|}
\hline \multirow{2}{*}{$\begin{array}{l}\text { Main focus } \\
\text { Pathological type }\end{array}$} & \multicolumn{2}{|c|}{ Minor focus } & \multirow{2}{*}{$\begin{array}{l}\text { Concordance } \\
\text { rate (\%) } \\
87.3\end{array}$} & \multirow{2}{*}{$\begin{array}{l}\text { Kappa } \\
0.633\end{array}$} & \multirow{2}{*}{$\begin{array}{l}\text { Pvalue } \\
\\
<0.001\end{array}$} \\
\hline & IDC & Non-IDC & & & \\
\hline IDC & 236 & 31 & & & \\
\hline Non-IDC & 11 & 52 & & & \\
\hline Histological grade & Q & प & 88.4 & 0.772 & $<0.001$ \\
\hline$\square$ & 15 & 0 & & & \\
\hline$\square$ & 13 & 5 & & & \\
\hline Q & 1 & 59 & & & \\
\hline ER & Negative & Positive & 97.0 & 0.915 & $<0.001$ \\
\hline Negative & 71 & 2 & & & \\
\hline Positive & 8 & 249 & & & \\
\hline PR & Negative & Positive & 93.9 & 0.867 & $<0.001$ \\
\hline Negative & 106 & 8 & & & \\
\hline Positive & 12 & 204 & & & \\
\hline HER2 & Negative & Positive & 96.5 & 0.906 & $<0.001$ \\
\hline Negative & 231 & 5 & & & \\
\hline Positive & 6 & 72 & & & \\
\hline Ki67 & $<20 \%$ & $\geq 20 \%$ & 91.2 & 0.846 & $<0.001$ \\
\hline$<20 \%$ & 140 & 7 & & & \\
\hline$\geq 20 \%$ & 22 & 161 & & & \\
\hline
\end{tabular}

The molecular subtypes were identical within the same patient in $276(88.2 \%)$ of the 313 cases using the 2013 St Gallen standard, with 72 luminal A-like tumors, 102 luminal B-like (HER2-) tumors, 36 luminal Blike (HER2+) tumors, 36 HER2-enriched tumors, and 30 triple negative breast cancers (Table 3). Molecular subtypes differed among different tumor foci in $37(11.8 \%)$ patients. 
Table 3

Concordance rates of molecular subtypes ${ }^{a}$

\begin{tabular}{|c|c|c|c|c|c|c|c|c|}
\hline \multirow{2}{*}{$\begin{array}{l}\text { Main } \\
\text { focus }\end{array}$} & \multicolumn{5}{|c|}{ Minor focus } & \multirow{2}{*}{$\begin{array}{l}\text { Concordance rate } \\
\text { (\%) } \\
88.2\end{array}$} & \multirow{2}{*}{$\begin{array}{l}\text { Kappa } \\
0.841\end{array}$} & \multirow{2}{*}{$\begin{array}{l}P \\
\text { value } \\
< \\
0.001\end{array}$} \\
\hline & LA & $\begin{array}{l}\text { LB } \\
\text { (HER2-) }\end{array}$ & $\begin{array}{l}\text { LB } \\
\text { (HER2+) }\end{array}$ & HER2+ & TNBC & & & \\
\hline LA & 72 & 7 & 0 & 2 & 0 & & & \\
\hline $\begin{array}{l}\text { LB } \\
\text { (HER2-) }\end{array}$ & 15 & 102 & 2 & 1 & 3 & & & \\
\hline $\begin{array}{l}\text { LB } \\
\text { (HER2+) }\end{array}$ & 1 & 3 & 36 & 0 & 0 & & & \\
\hline HER2+ & 0 & 0 & 0 & 36 & 2 & & & \\
\hline TNBC & 1 & 0 & 0 & 0 & 30 & & & \\
\hline
\end{tabular}

a : The cut-off value of Ki67 was $20 \%$ for differentiating Luminal A-like and Luminal B-like (HER2-).

Abbreviations: HER2, human epidermal growth factor receptor 2; HR, hormone receptor; LA, Luminal Alike; LB, Luminal B-like; TNBC, triple negative breast cancer.

\section{Heterogeneity of molecular markers and adjuvant therapy}

A total of $45(84.9 \%)$ patients in the Hetero group received adjuvant endocrine therapy, which was significantly higher than that of patients in the Homo group $(71.7 \%, P=0.046$, Fig. $2 \mathrm{~A})$. There were no significant differences in the usage rates of adjuvant anti-HER2 therapy $(28.3 \%$ versus [vs.] $19.6 \%, P=$ 0.196, Fig. 2B) and chemotherapy (69.9\% vs. 69.8\%, $P=0.987$, Fig. 2C) between the two groups.

\section{Heterogeneity of molecular markers and disease outcomes}

At a median follow-up time of 35 (IQR: 19-57) months, 21 DFS events were recorded: 16 in the Homo group and 5 in the Hetero group (Table 4). As shown in Table 5, status of molecular markers was significantly associated with DFS $(P=0.041)$. The estimated 3 -year DFS rate was $81.2 \%$ for the Hetero group, which was significantly higher than that of the Homo group $(96.5 \%, H R=2.81,95 \% \mathrm{Cl}$ : 1.00-7.88, Fig. 3A). Two patients in the Hetero group and 5 in the Homo group died during the follow-up period (Table 4). Patients in the hetero group tended to have shorter OS, with the estimated 3-year OS rates for these two groups being $95.8 \%$ and $99.5 \%$, respectively $(H R=4.31,95 \% \mathrm{Cl}: 0.83-22.46, P=0.059, \mathrm{Fig} .3 \mathrm{~B})$. 
Table 4

Details of DFS and OS events by status of molecular markers

\begin{tabular}{|llll|}
\hline & Total & Homo & Hetero \\
& $\mathbf{N = 3 3 0 ( \% )}$ & $\mathbf{N = 2 7 7 ( \% )}$ & $\mathbf{N}=\mathbf{5 3}(\%)$ \\
\hline DFS events & & & \\
\hline No recurrence & $309(93.6)$ & $261(94.2)$ & $48(90.6)$ \\
\hline Local-regional recurrence & $5(1.5)$ & $4(1.4)$ & $1(1.9)$ \\
\hline Contralateral breast cancer & $4(1.2)$ & $4(1.4)$ & $0(0.0)$ \\
\hline Second non-breast malignancy & $2(0.6)$ & $1(0.4)$ & $1(1.9)$ \\
\hline Distant recurrence & $8(2.4)$ & $6(2.2)$ & $2(3.8)$ \\
\hline Death without recurrence & $2(0.6)$ & $1(0.4)$ & $1(1.9)$ \\
\hline OS events & & & \\
\hline Alive & $323(97.9)$ & $272(98.2)$ & $51(96.2)$ \\
\hline Death of any cause & $7(2.1)$ & $5(1.8)$ & $2(3.9)$ \\
\hline Death with recurrence & $5(1.5)$ & $4(1.4)$ & $1(1.9)$ \\
\hline Death without recurrence & $2(0.6)$ & $1(0.4)$ & $1(1.9)$ \\
\hline
\end{tabular}


Table 5

Univariate analysis of prognostic factors affecting DFS and OS in multiple breast cancer

\begin{tabular}{|c|c|c|}
\hline \multirow[t]{2}{*}{ Characteristics } & \multicolumn{2}{|c|}{$P$ value } \\
\hline & DFS & OS \\
\hline Age $(<50 \mathrm{y} / \mathrm{o} v s . \geq 50 \mathrm{y} / \mathrm{o})$ & 0.741 & 0.277 \\
\hline Menstrual status (Pre/Peri- vs. Post-) & 0.966 & 0.969 \\
\hline Number of foci (2 vs. 3/4) & 0.264 & 0.557 \\
\hline Histology type (IDC vs. non-IDC) ${ }^{a}$ & 0.802 & 0.789 \\
\hline Tumor size $(\leq 2.0 \mathrm{~cm} v s .>2.0 \mathrm{~cm})^{a}$ & 0.786 & 0.292 \\
\hline ALN status (Negative vs. Positive) & 0.572 & 0.841 \\
\hline Histological grade $(\llbracket v s . \otimes v s . \otimes v s . N A)^{a}$ & 0.637 & 0.892 \\
\hline LVI (Negative vs. Positive) & 0.162 & 0.512 \\
\hline Group (Homo vs. Hetero) & 0.041 & 0.059 \\
\hline
\end{tabular}

a : main focus.

Abbreviations: ALN, axillary lymph node; IDC: invasive ductal carcinoma; DFS: disease-free survival; LVI, lymph-vascular invasion; NA: not available; OS: overall survival; y/o, years old.

\section{Discussion}

The study was designed to evaluate the rates of molecular markers heterogeneity as well as its associations with systemic adjuvant therapy and disease outcomes in MMBC. Molecular markers showed good concordance among different invasive tumor foci. Heterogeneity of ER, PR, HER2, and Ki67 were present in $\mathrm{MMBC}$, which was associated with more adjuvant endocrine therapy usage $(P=0.046)$ and shorter DFS $(P=0.041)$, indicating the necessity of molecular assessments for different tumor foci in patients with MMBC.

There were some published literatures that reported the rates of intratumor biomarkers heterogeneity among different foci in MMBC [13, 22-25]. For example, Buggi and colleagues enrolled 113 invasive multiple breast cancers and they reported mismatches on ER, PR, HER2, and Ki67 in 4.4\%, 15.9\%, 9.7\%, and $15.0 \%$ cases [13]. Similarly, the rate of ER, PR, HER2, and Ki67 heterogeneity in our cohort was 3.0\%, $6.1 \%, 3.5 \%$, and $8.8 \%$ respectively. Moreover, molecular subtypes differed in $37(11.8 \%)$ patients as classified by 2013 St Gallen system, comparable to the results of Pekar et al (12.7\%) [14].

With limited knowledge on the consequences of molecular heterogeneity for therapeutic decision-making, it has been accepted that biomarkers can be assessed only in the largest individual tumor focus [26]. This is based on the observations that molecular markers in MMBC are usually homogenous. However, if the 
tumor foci demonstrate different pathological or histological features, biomarkers evaluation of the smaller focus will be necessary. According to Buggi et al, 14 out of the $113(12.4 \%)$ patients received additional systemic treatments with the biomarkers analysis for the smaller tumor focus [13]. In the present study, heterogeneity of biomarkers was also found to be significantly associated with the usage of adjuvant endocrine therapy.

To the best of the authors' knowledge, this was the largest study to evaluate the prognostic significance of intratumor biomarkers heterogeneity. Our cohort revealed that patients in the Hetero group had significantly shorter DFS $(H R=2.81,95 \% \mathrm{Cl}$ : $1.00-7.88, P=0.041)$ and marginally worse OS $(H R=4.31$, $95 \% \mathrm{Cl}: 0.83-22.46, P=0.059)$ compared to patients in the Homo group. Consistent with our results, Pekar et al concluded that patients with phenotypically heterogenous MMBC had a significantly shorter breast cancer specific survival $(H R=2.87,95 \% \mathrm{Cl}: 1.08-7.64, P=0.034)$ and overall survival $(H R=2.80$, $95 \% \mathrm{Cl}: 1.05-7.44, P=0.039)$ [14]. Taken together, these results suggested the necessity of evaluating molecular markers for different tumor foci in patients with $M M B C$ [27]. And it would strengthen our point if we can verify that patients with multicentric disease who do not undergo a second biopsy have worse outcome. However, only 3 out of 30 patients with multiple invasive diseases who lacked biomarkers data in our cohort experienced DFS events, indicating a large cohort with more patients are needed to validation this recommendation.

The present study enrolled both multifocal and multicentric diseases, which were heterogenous and could be further differentiated into multifocal breast cancer (MFBC) and multicentric breast cancer (MCBC). In our cohort, ER status, PR status, and breast surgery were significantly different between patients with MFBC and MCBC (Supplementary Table S1, S2). While for DFS and OS, no significant differences were observed between the two groups (Supplementary Figure S4). To date, relatively few reports have directly compared the clinical-pathological features, treatment patterns and survivals of patients with MFBC and $M C B C$, which warrant further research [28-30].

The incidence of MMBC increases with the advancement of preoperative imaging, and intratumor molecular heterogeneity has attracted the attention of clinicians. For example, MRI can identify $74.6 \%$, $54.2 \%$, and $67.3 \%$ of MMBC that were not identified by physical examination, sonography, or mammography, respectively (Supplementary Figure S1). We performed the study for the first time to evaluate the rates of biomarkers heterogeneity in $\mathrm{MMBC}$ as well as its impacts on adjuvant therapy and survival. However, there were several limitations in the present study. Firstly, this was a single-institutional retrospective study, so there might be selection bias and limited applicability. Further validation in other cohorts will provide us more insights to the therapeutic and prognostic role of biomarkers heterogeneity. Secondly, the Hetero group consisted of a relatively small number of patients and cases, which warranted longer follow-up. Thirdly, the present study enrolled patients over a nine-year period from Jan 2009 through Dec 2018, which might exert an influence to disease outcomes. However, no significant disease outcome difference was observed between patients who received surgery before or after 2015 in our cohort (Supplementary Figure S5). 
In conclusion, heterogeneity of ER, PR, HER2, or Ki67 was present in $16.1 \%$ patients with MMBC. Biomarkers heterogeneity was associated with more adjuvant endocrine therapy usage and worse disease outcomes, indicating the necessity of molecular assessments for different tumor foci in patients with MMBC.

\section{Declarations}

\section{Funding}

This study was funded by the National Natural Science Foundation of China (Grant Number: 81772797). Shanghai Municipal Education Commission-Gaofeng Clinical Medicine Grant Support (20172007); Ruijin Hospital, Shanghai Jiao Tong University School of Medicine-"Guangci Excellent Youth Training Program" (GCQN-2017-A18). All these financial sponsors had no role in the study design, data collection, analysis or interpretation.

\section{Conflict of interest}

The authors declare no conflict of interest.

\section{Availability of data and material}

The datasets used and/or analysed during the current study are available from the corresponding author on reasonable request.

\section{Code availability}

Not appliable.

\section{Authors' contributions}

XC conceived and designed the study. SL analyzed and interpreted the data for presentation and was a main contributor in writing the manuscript. XC revised the manuscript and all authors approved the final manuscript.

\section{Ethical approval}

This article does not contain any studies with human participants performed by any of the authors. All procedures performed in studies involving human participants were in accordance with the ethical standards of the institutional and/or national research committee and with the 1964 Helsinki declaration and its later amendments or comparable ethical standards.

\section{Consent to participate}

Not appliable. 


\section{Consent for publication}

Not appliable.

\section{Acknowledgements}

Not appliable.

\section{References}

1. Zardavas D, Irrthum A, Swanton C, et al. Clinical management of breast cancer heterogeneity. Nat Rev Clin Oncol 2015; 12(7): 381-94.

2. Goldhirsch A, Wood WC, Coates AS, et al. Strategies for subtypes-dealing with the diversity of breast cancer: highlights of the St. Gallen International Expert Consensus on the Primary Therapy of Early Breast Cancer 2011. Ann Oncol 2011; 22(8): 1736-47.

3. Aurilio G, Disalvatore D, Pruneri G, et al. A meta-analysis of oestrogen receptor, progesterone receptor and human epidermal growth factor receptor 2 discordance between primary breast cancer and metastases. Eur J Cancer 2014; 50(2): 277-89.

4. Yates LR. Intratumoral heterogeneity and subclonal diversification of early breast cancer. Breast 2017; 34 Suppl 1: S36-S42.

5. Yeo SK, Guan JL. Breast Cancer: Multiple Subtypes within a Tumor? Trends Cancer 2017; 3(11): 75360.

6. Falck AK, Bendahl PO, Chebil G, et al. Biomarker expression and St Gallen molecular subtype classification in primary tumours, synchronous lymph node metastases and asynchronous relapses in primary breast cancer patients with 10 years' follow-up. Breast Cancer Res Treat 2013; 140(1): 93104.

7. Corso G, Magnoni F, Provenzano E, et al. Multicentric breast cancer with heterogeneous histopathology: a multidisciplinary review. Future Oncol 2020; 16(8): 395-412.

8. Ng CK, Martelotto LG, Gauthier A, et al. Intra-tumor genetic heterogeneity and alternative driver genetic alterations in breast cancers with heterogeneous HER2 gene amplification. Genome Biol 2015; 16: 107.

9. Muller KE, Marotti JD, Tafe LJ. Pathologic Features and Clinical Implications of Breast Cancer with HER2 Intratumoral Genetic Heterogeneity. Am J Clin Pathol 2019; 152(1): 7-16.

10. Burrell RA, McGranahan N, Bartek J, et al. The causes and consequences of genetic heterogeneity in cancer evolution. Nature 2013; 501(7467): 338-45.

11. Gerlinger M, Rowan AJ, Horswell S, et al. Intratumor heterogeneity and branched evolution revealed by multiregion sequencing. N Engl J Med 2012; 366(10): 883-92.

12. Dagogo-Jack I, Shaw AT. Tumour heterogeneity and resistance to cancer therapies. Nat Rev Clin Oncol 2018; 15(2): 81-94. 
13. Buggi F, Folli S, Curcio A, et al. Multicentric/multifocal breast cancer with a single histotype: is the biological characterization of all individual foci justified? Ann Oncol 2012; 23(8): 2042-6.

14. Pekar G, Gere M, Tarjan M, et al. Molecular phenotype of the foci in multifocal invasive breast carcinomas: intertumoral heterogeneity is related to shorter survival and may influence the choice of therapy. Cancer 2014; 120(1): 26-34.

15. Chen $X$, Sun $L$, Mao Y, et al. Preoperative core needle biopsy is accurate in determining molecular subtypes in invasive breast cancer. BMC Cancer 2013; 13: 390.

16. Zhu S, Wu J, Huang O, et al. Clinicopathological Features and Disease Outcome in Breast Cancer Patients with Hormonal Receptor Discordance between Core Needle Biopsy and Following Surgical Sample. Ann Surg Oncol 2019; 26(9): 2779-86.

17. Hammond ME, Hayes DF, Dowsett M, et al. American Society of Clinical Oncology/College of American Pathologists guideline recommendations for immunohistochemical testing of estrogen and progesterone receptors in breast cancer. J Clin Oncol 2010; 28(16): 2784-95.

18. Wolff AC, Hammond ME, Schwartz JN, et al. American Society of Clinical Oncology/College of American Pathologists guideline recommendations for human epidermal growth factor receptor 2 testing in breast cancer. J Clin Oncol 2007; 25(1): 118-45.

19. Wolff AC, Hammond ME, Hicks DG, et al. Recommendations for human epidermal growth factor receptor 2 testing in breast cancer: American Society of Clinical Oncology/College of American Pathologists clinical practice guideline update. J Clin Oncol 2013; 31(31): 3997-4013.

20. Wolff AC, Hammond MEH, Allison KH, et al. Human Epidermal Growth Factor Receptor 2 Testing in Breast Cancer: American Society of Clinical Oncology/College of American Pathologists Clinical Practice Guideline Focused Update. J Clin Oncol 2018; 36(20): 2105-22.

21. Tong $Y$, Chen $X$, Fei $X$, et al. Can breast cancer patients with HER2 dual-equivocal tumours be managed as HER2-negative disease? Eur J Cancer 2018; 89: 9-18.

22. Garimella V, Long ED, O'Kane SL, et al. Oestrogen and progesterone receptor status of individual foci in multifocal invasive ductal breast cancer. Acta Oncol 2007; 46(2): 204-7.

23. Choi Y, Kim EJ, Seol H, et al The hormone receptor, human epidermal growth factor receptor 2, and molecular subtype status of individual tumor foci in multifocal/multicentric invasive ductal carcinoma of breast. Hum Pathol 2012; 43(1): 48-55.

24. Boros M, llyes A, Nechifor Boila A, et al. Morphologic and molecular subtype status of individual tumor foci in multiple breast carcinoma. A study of 155 cases with analysis of 463 tumor foci. Hum Pathol 2014; 45(2):409-416.

25. Singh K, Wang Y, Marketkar S, et al. Comparison of Estrogen Receptor, Progesterone Receptor and HER2 Results in Concurrent Ipsilateral Samples with Invasive Breast Carcinoma; A Retrospective Study of 246 Biopsies From 119 Patients. Hum Pathol 2017; 65: 123-132.

26. Lester SC, Bose S, Chen YY, et al. Protocol for the examination of specimens from patients with invasive carcinoma of the breast. Arch Pathol Lab Med 2009; 133(10): 1515-38. 
27. Pooja N, Bleiweiss IJ, Shabnam J, et al. Evaluation of Biomarkers in Multiple Ipsilateral Synchronous Invasive Breast Carcinomas. Arch Patho Lab Med 2019; 143(2):190-196.

28. Lynch SP, Lei $X$, Chavez-Macgregor M, et al. Multifocality and multicentricity in breast cancer and survival outcomes. Ann Oncol 2012; 23(12):3063-3069.

29. Kadioğlu H, Özbas S, Akcan A, et al. Comparison of the histopathology and prognosis of bilateral versus unilateral multifocal multicentric breast cancers. World J Surg Oncol 2014; 12(1):266-274.

30. Ataseven B, Lederer B, Blohmer JU, et al. Impact of Multifocal or Multicentric Disease on Surgery and Locoregional, Distant and Overall Survival of 6,134 Breast Cancer Patients Treated With Neoadjuvant Chemotherapy. Ann Surg Oncol, 2015; 22(4):1118-1127.

\section{Figures}

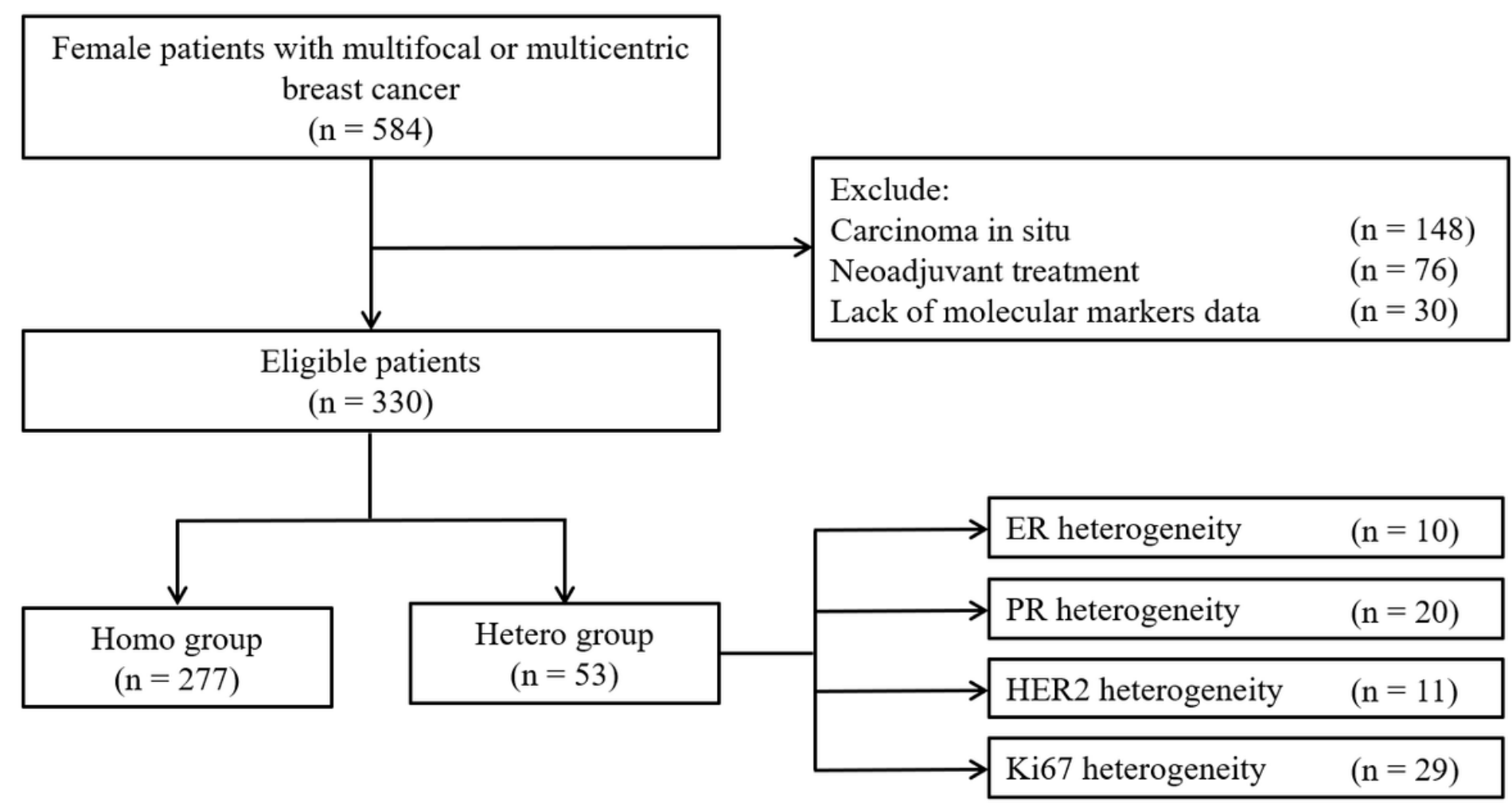

Figure 1

Folw chart of 330 patients in the study 
A

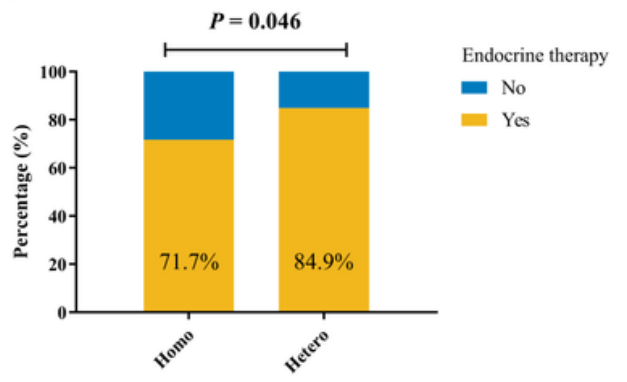

B

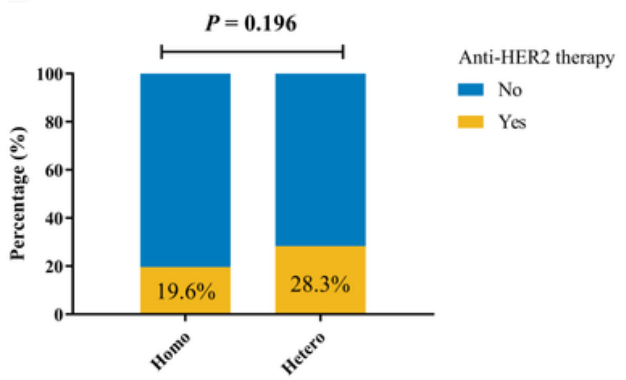

C

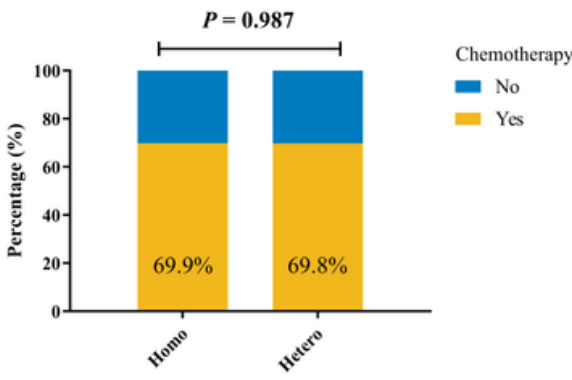

Figure 2

Adjuvant systemic therapy by molecular markers status

A

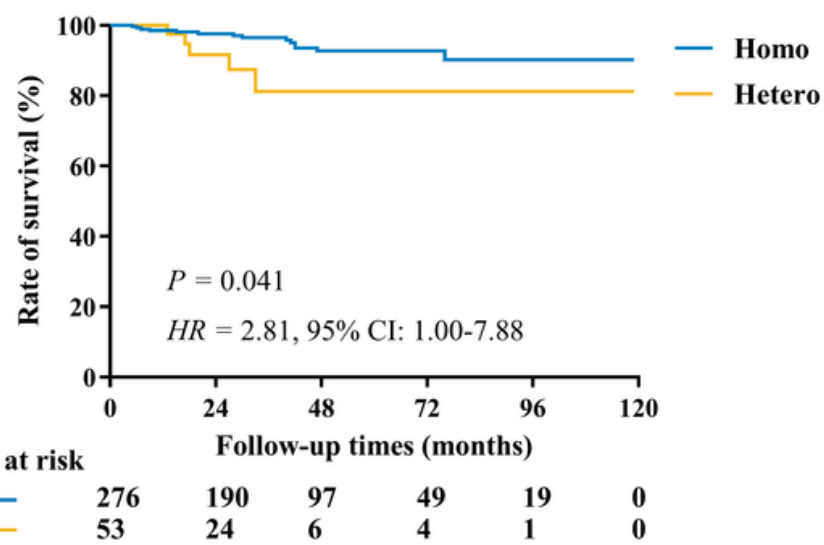

B

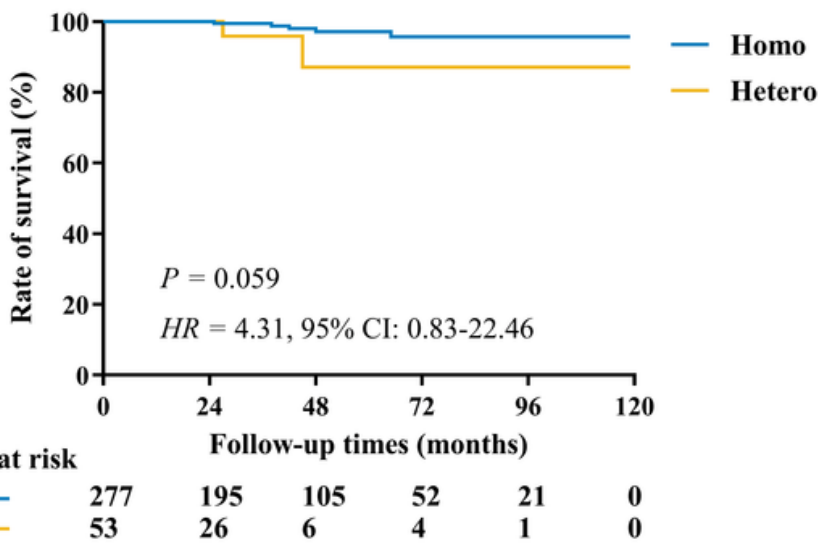

\section{Figure 3}

Kaplan-Meier curves of DFS and OS by molecular markers status. (A) The estimated 3-year DFS rates for the Homo and Hetero groups were $96.5 \%$ and $81.2 \%$, respectively $(P=0.041)$. (B) The estimated 3-year OS rates for the Homo and Hetero groups were $99.5 \%$ and $95.8 \%$, respectively $(P=0.059)$. 